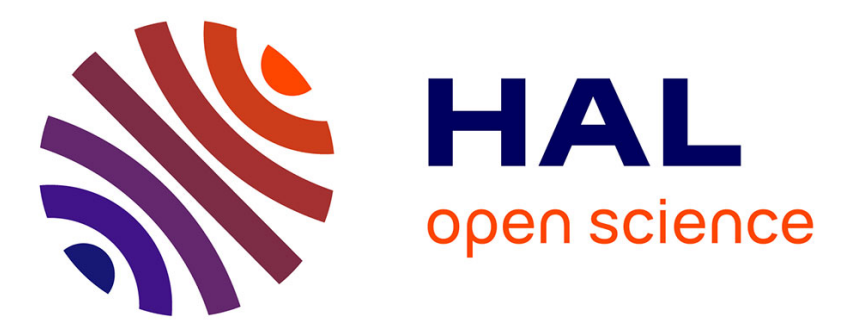

\title{
Acoustical measurement of the shear modulus for thin porous layers
}

\author{
Jean-François Allard, Michel Henry, Laurens Boeckx, Philippe Leclaire, \\ Walter Lauriks
}

\section{- To cite this version:}

Jean-François Allard, Michel Henry, Laurens Boeckx, Philippe Leclaire, Walter Lauriks. Acoustical measurement of the shear modulus for thin porous layers. Journal of the Acoustical Society of America, 2005, 117 (4), pp.1737. 10.1121/1.1868392 . hal-01326764

\section{HAL Id: hal-01326764 \\ https://hal.science/hal-01326764}

Submitted on 5 Jun 2016

HAL is a multi-disciplinary open access archive for the deposit and dissemination of scientific research documents, whether they are published or not. The documents may come from teaching and research institutions in France or abroad, or from public or private research centers.
L'archive ouverte pluridisciplinaire HAL, est destinée au dépôt et à la diffusion de documents scientifiques de niveau recherche, publiés ou non, émanant des établissements d'enseignement et de recherche français ou étrangers, des laboratoires publics ou privés. 


\title{
Acoustical measurement of the shear modulus for thin porous layers
}

\author{
Jean F. Allard and Michel Henry \\ Laboratoire d'Acoustique de l'Université du Maine, UMR CNRS 6613, Avenue Olivier Messiaen, \\ 72085 Le Mans Cedex, France \\ Laurens Boeckx, Philippe Leclaire, and Walter Lauriks \\ Laboratorium voor Akoestiek en Thermische Fysica, Departement Natuurkunde, Katholieke Universiteit \\ Leuven, Celestijnenlaan 200 D, B-3001 Heverlee, Belgium
}

\begin{abstract}
Simulations performed with the Biot theory show that for thin porous layers, a shear mode of the structure can be induced by a point-source in air located close to the layer. The simulations show that this mode is present around frequencies where the quarter wavelength of the shear Biot wave is equal to the thickness of the samples and show that it can be acoustically detected from the fast variations with frequency of the location of a pole of the reflection coefficient close to grazing incidence. The mode has been detected with this method for two reticulated plastic foams. For one of the foams studied, the velocity and the damping of the Rayleigh wave have been measured on a thicker layer of the same medium at higher frequencies, giving a real part of the shear modulus close to the one obtained from the measured location of the pole. The strong coupling of the shear mode with the acoustic field in air allows the measurement of the shear modulus without mechanical excitation.
\end{abstract}

\section{INTRODUCTION}

Acoustical excitations from a sound source in air generally do not create noticeable displacements of the porous frame for usual sound-absorbing materials. As a consequence, the measurement of the rigidity coefficients of the porous frames is performed with mechanical excitations. ${ }^{1-7}$ A contact surface with a static stress is generally present and the material, often strongly nonlinearly reacting, can be modified by the experimental procedure. This drawback is avoided when the Rayleigh wave velocity is measured ${ }^{8}$ but this measurement can only be performed on sufficiently thick layers; the penetration of the Rayleigh wave inside the material is of several shear wavelengths. Free field measurements of the surface impedance can be used, with the Biot theory, ${ }^{9,10}$ to evaluate the velocity of the frame-borne compressional wave. ${ }^{11}$ At normal incidence, a mode related to this wave can be excited. This mode is with a good approximation the quarter wavelength resonance of the compressional wave in the frame, and is present at frequencies where the wavelength is close to four times the thickness $l$ of the layer. The surface impedance at normal incidence for a layer glued to a rigid impervious backing presents around these frequencies fast variations if the loss angle of the frame is sufficiently small, but this is not the case for most of the porous sound absorbing media. The excitation of shear modes related to the Biot shear wave would need less energy at equal loss angles because the shear modulus is smaller than the modulus related to frame compressional waves. These modes cannot be excited by plane waves at normal incidence but at large angles of incidence $\theta$. At $\theta=\pi / 2$, the surface impedance $Z_{s}(\pi / 2)$ can be measured with the Tamura method, ${ }^{12}$ but areas as large as $10 \mathrm{~m}^{2}$ are needed, over which the porous layer must be carefully glued to a rigid impervious backing, and the experimental procedure is complicated. It has been shown recently that for thin porous layers with a motionless frame (the thickness $l$ of a thin layer verifies $|\delta| l \ll 1$, where $\delta$ is the wave number in the air saturating the frame), a pole of the reflection coefficient exists at a complex angle of incidence $\theta_{p}$ close to $\pi / 2$, and $\theta_{p}$ can be evaluated easily from measurements of the monopole pressure field close to grazing incidence. ${ }^{13-15}$ More precisely, the reflection coefficient $V$ is given by

$$
V(\cos \theta)=\frac{Z_{s}(\cos \theta)-Z / \cos \theta}{Z_{S}(\cos \theta)+Z / \cos \theta},
$$

and the angle $\theta_{p}$ is a solution of the following equation

$$
\cos \theta_{p}=-\frac{Z}{Z_{s}\left(\cos \theta_{p}\right)},
$$

where $Z$ is the characteristic impedance of air, and the surface impedance $Z_{s}$ is given by

$$
Z_{s}(\theta)=\frac{i Z_{1}}{\phi \cos \theta_{1}} \cot \left(\delta l \cos \theta_{1}\right) .
$$

In this equation, $Z_{1}$ is the characteristic impedance in the fluid equivalent to the air saturating the porous medium, $\phi$ is the porosity, and $\theta_{1}$ is the refraction angle given by $\sin \theta_{1}$ $=(\sin \theta) k / \delta, k$ being the wave number in the free air. For thin layers $\theta_{p}$ is close to $\pi / 2$ because $Z /\left|Z_{s}\left(\theta_{p}\right)\right| \ll 1$, and $\cos \theta_{p}$ is close to $-Z / Z_{s}(\pi / 2)$. In Fig. 1, a sketch of the experimental set for the measurement method is presented. The monopole 


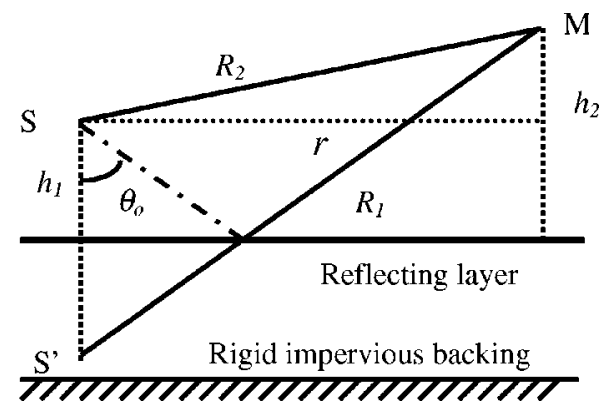

FIG. 1. Symbolic sketch of the experiment, the monopole source S, and the receiver at $\mathrm{M}$ above the porous layer.

source $\mathrm{S}$ and the receiver at $\mathrm{M}$ are located at small distances, respectively $h_{1}$ and $h_{2}$, from the porous layer. The distance $R_{2}$ from the source to the receiver, $R_{1}$ from the image of the source to the receiver, and the radial distance $r$ are much larger than $h_{1}+h_{2}$, and the angle of specular incidence $\theta_{0}$ is close to $\pi / 2$. (The symbols $\theta_{0}$ and $\theta$ are used for the angle of specular incidence and for the angle of incidence of plane waves, respectively.) The method is based on the use of the following approximation for the monopole reflected field $p_{r}$ over thin porous layers with a motionless frame, valid under the condition $\theta_{0}$ and $\theta_{p}$ close to $\pi / 2$ :

$$
\begin{aligned}
p_{r}= & \frac{\exp \left(i k R_{1}\right)}{R_{1}}\left[1-\sqrt{2 \pi k R_{1}} \exp \left(\frac{3 \pi i}{4}\right)\right. \\
& \left.\times \cos \theta_{p} \exp \left(-w^{2}\right) \operatorname{erfc}(-i w)\right], \\
w= & \sqrt{2 k R_{1}} \exp \frac{\pi i}{4} \sin \frac{\theta_{p}-\theta_{0}}{2} .
\end{aligned}
$$

Under the condition $\theta_{0}$ and $\theta_{p}$ close to $\pi / 2, w$ can be rewritten

$$
w=\sqrt{\frac{1}{2} k R_{1}} \frac{\pi i}{4} \exp \left(\cos \theta_{0}-\cos \theta_{p}\right) .
$$

The possible variations of $Z_{s}\left(\theta_{p}\right)$ around frequencies where the shear mode is excited are much easier to measure with the new method than $Z_{s}(\pi / 2)$ with the Tamura method. The Biot theory is used in Sec. II to describe the shear mode and to predict the surface impedance and $\cos \theta_{p}$. The validity of Eqs. (4)-(6) in the context of the Biot theory is discussed in Sec. III. Measurements of $\cos \theta_{p}$ leading to an evaluation of the shear modulus for two porous foams are presented in Sec. IV.

\section{DESCRIPTION OF THE SHEAR MODE}

The Biot theory, with the formalism developed in Ref. 16 , has been used to predict the surface impedance with the model by Johnson et al. ${ }^{17}$ for the viscous and inertial interaction, and the model by Lafarge ${ }^{18}$ for the incompressibility of air. The stress-strain relations in the Biot theory are

$$
\begin{aligned}
\sigma_{i j}^{s} & =\left[(P-2 N) \theta^{s}+Q \theta^{f}\right] \delta_{i j}+2 N e_{i j}^{s}, \\
\sigma_{i j}^{f} & =Q \theta^{s}+R \theta^{f},
\end{aligned}
$$

where $\theta^{f}$ and $\theta^{s}$ are the dilatation of the air and of the frame, respectively, $e_{i j}^{s}$ are the strain components of the frame, and $\sigma_{i j}^{f}$ and $\sigma_{i j}^{s}$ are the stress components of the air and of the frame, respectively. The stress components are related to forces per unit area of porous medium; then $\sigma_{i j}^{f}=-\phi p \delta_{i j}$, where $p$ is the pressure in the air that saturates the porous medium. The Biot elasticity coefficients $P, Q$, and $R$, with the simplifications suggested in Ref. 16 (see Eqs. 6.21-6.28 of Ref. 16), are given by

$$
\begin{aligned}
& P=\frac{4}{3} N+K_{b}+\frac{(1-\phi)^{2}}{\phi} K_{f}, \\
& Q=K_{f}(1-\phi), \\
& R=\phi K_{f}, \\
& K_{b}=\frac{2}{3} N(1+\nu) /(1-2 \nu),
\end{aligned}
$$

where $K_{b}$ is the bulk modulus of the frame, $N$ is the shear modulus, and $\nu$ is the Poisson ratio. The incompressibility $K_{f}$ of the air saturating the porous frame is given in the present work by

$$
K_{f}=\frac{K_{a}}{\beta},
$$

where $K_{a}$ is the adiabatic incompressibility of air and $\beta$ is given by Eq. (30) of Ref. 18:

$$
\begin{aligned}
& \beta=\gamma-(\gamma-1)\left[1+\frac{1}{-i \widetilde{\omega}^{\prime}}\left(1-\frac{M^{\prime}}{2} i \widetilde{\omega}^{\prime}\right)^{1 / 2}\right]^{-1}, \\
& \widetilde{\omega}^{\prime}=\frac{\rho_{0} \operatorname{Pr} \omega k_{0}^{\prime}}{\eta \phi}, \\
& M^{\prime}=\frac{8 k_{0}^{\prime}}{\phi \Lambda^{\prime 2}} .
\end{aligned}
$$

In these equations, $\eta$ is the viscosity, $\rho_{0}$ is the density of air, $\gamma$ is the ratio of the specific heats, $\operatorname{Pr}$ is the Prandtl number, $k_{0}^{\prime}$ is the thermal permeability, and $\Lambda^{\prime}$ is the thermal characteristic dimension of the porous frame.

The equations of motion governing the displacement $\mathbf{u}^{s}$ of the frame and $\mathbf{u}^{f}$ of the saturating fluid are

$$
\begin{aligned}
-\omega^{2}\left(\tilde{\rho}_{11} \mathbf{u}^{s}+\tilde{\rho}_{12} \mathbf{u}^{f}\right)= & (P-N) \boldsymbol{\nabla} \boldsymbol{\nabla} \cdot \mathbf{u}^{s}+N \nabla^{2} \mathbf{u}^{s} \\
& +Q \boldsymbol{\nabla} \boldsymbol{\nabla} \cdot \mathbf{u}^{f}, \\
-\omega^{2}\left(\tilde{\rho}_{22} \mathbf{u}^{f}+\tilde{\rho}_{12} \mathbf{u}^{s}\right)= & R \boldsymbol{\nabla} \boldsymbol{\nabla} \cdot \mathbf{u}^{f}+Q \boldsymbol{\nabla} \boldsymbol{\nabla} \cdot \mathbf{u}^{s} .
\end{aligned}
$$

The renormalized densities $\tilde{\rho}_{11}, \tilde{\rho}_{22}$, and $\tilde{\rho}_{12}$ are given by (see Eqs. 6.58 of Ref. 16)

$$
\begin{aligned}
& \tilde{\rho}_{11}=\rho_{1}+\rho_{a}-i b, \\
& \tilde{\rho}_{12}=-\rho_{a}+i b, \\
& \tilde{\rho}_{22}=\phi \rho_{0}+\rho_{a}-i b .
\end{aligned}
$$

The coefficient $b$ related to the viscous and the inertial interaction is given in the present work by

$$
b=-\frac{\sigma}{\omega} \phi^{2} G_{j}(\omega)
$$




$$
G_{j}(\omega)=\left(1-\frac{4 i \alpha_{\infty}^{2} \eta \rho_{0} \omega}{\sigma^{2} \Lambda^{2} \phi^{2}}\right)^{1 / 2}
$$

(see Eq. 5-64 of Ref. 16 with a complex conjugation due to the opposite time dependence), where $\alpha_{\infty}$ is the tortuosity, $\sigma$ is the flow resistivity, and $\Lambda$ is the characteristic viscous dimension. The added density $\rho_{a}$ is given by

$$
\rho_{a}=\rho_{0} \phi\left(\alpha_{\infty}-1\right) .
$$

The wave numbers $k_{1}$ and $k_{2}$ for both Biot compressional waves, and $k_{3}$ for the Biot shear wave, are given by

$$
\begin{aligned}
k_{1,2}^{2}= & \frac{\omega^{2}}{2\left(P R-Q^{2}\right)}\left[P \tilde{\rho}_{22}+R \tilde{\rho}_{11}-2 Q \tilde{\rho}_{12} \pm \sqrt{\Delta}\right], \\
k_{3}^{2}= & \frac{\omega^{2}}{N}\left[\frac{\tilde{\rho}_{11} \tilde{\rho}_{22}-\tilde{\rho}_{12}^{2}}{\tilde{\rho}_{22}}\right], \\
\Delta= & \left(P \tilde{\rho}_{22}+R \tilde{\rho}_{11}-2 Q \tilde{\rho}_{12}\right)^{2} \\
& -4\left(P R-Q^{2}\right)\left(\tilde{\rho}_{11} \tilde{\rho}_{22}-\tilde{\rho}_{12}^{2}\right) .
\end{aligned}
$$

Both compressional waves can be related to a velocity potential $\varphi$ for the frame and $\varphi_{f}=\mu \varphi$ for the saturating fluid. The related displacements $\mathbf{u}^{s}$ and $\mathbf{u}^{f}$ are given by

$$
\begin{aligned}
& \mathbf{u}^{s}=\frac{i}{\omega} \nabla \varphi, \\
& \mathbf{u}^{f}=\frac{i \mu}{\omega} \nabla \varphi .
\end{aligned}
$$

The coefficients $\mu$ are given by

$$
\mu_{1,2}=\frac{P k_{1,2}^{2}-\omega^{2} \tilde{\rho}_{11}}{\omega^{2} \tilde{\rho}_{12}-Q k_{1,2}^{2}} .
$$

The shear wave is related to a vector velocity potential for the frame $\boldsymbol{\Psi}$, and a vector velocity potential for air $\boldsymbol{\Psi}_{a}$ $=\mu_{3} \Psi$.

The related displacements are given by

$$
\begin{aligned}
& \mathbf{u}^{s}=\frac{i}{\omega} \nabla \wedge \boldsymbol{\Psi}, \\
& \mathbf{u}^{f}=\frac{i}{\omega} \mu_{3} \boldsymbol{\nabla} \wedge \boldsymbol{\Psi},
\end{aligned}
$$

and $\mu_{3}$ is given by

$$
\mu_{3}=-\tilde{\rho}_{12} / \tilde{\rho}_{22} \text {. }
$$

A plane wave in air impinging upon the porous layer with an angle of incidence $\theta$ is represented in Fig. 2. The incidence plane is $x o z$, the axis $x$ is parallel to the air-porous medium interface. The incident field creates in the porous layer a field which can be described by six potentials with the same horizontal wave number vector component $\xi=k \sin \theta, \varphi_{1}^{+}, \varphi_{1}^{-}$ for the first compressional wave with opposite $z$ wave numbers vector components $\alpha_{1}=\left(k_{1}^{2}-\xi^{2}\right)^{1 / 2}$ and $-\alpha_{1}$, respectively. Similarly, two potentials $\varphi_{2}^{+}$and $\varphi_{2}^{-}$are defined for the second compressional wave, related to the $z$ wave number vector components $\alpha_{2}=\left(k_{2}^{2}-\xi^{2}\right)^{1 / 2}$ and $-\alpha_{2}$, and two potentials $\boldsymbol{\Psi}^{+}=\mathbf{n} \varphi_{3}^{+}, \quad \boldsymbol{\Psi}^{-}=\mathbf{n} \varphi_{3}^{-}$, related to $\alpha_{3}=\left(k_{3}^{2}\right.$

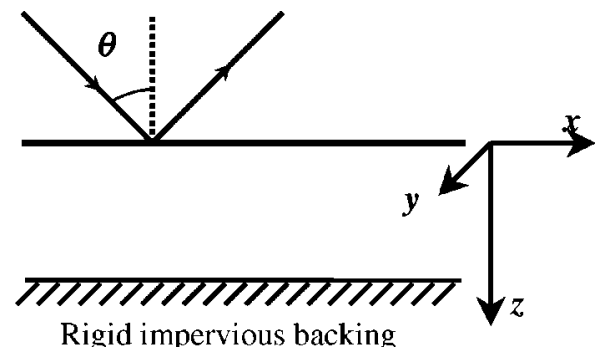

FIG. 2. A plane wave in air, incident on a porous layer. The angle of incidence is $\theta$, the incidence plane is $o x z$.

$\left.-\xi^{2}\right)^{1 / 2}$ and $-\alpha_{3}$, $\mathbf{n}$ being the unit vector on the $y$ axis. The scalar functions $\varphi_{j}^{ \pm}$can be written

$$
\varphi_{j}^{ \pm}=a_{j}^{ \pm} \exp \left( \pm i \alpha_{j} z+i \xi x\right)
$$

where the six constant coefficients $a_{j}^{ \pm}$can be predicted from the boundary conditions.

The porous layer is glued to the rigid impervious backing. At the contact surface with the rigid impervious backing the boundary conditions are $u_{z}^{s}=u_{x}^{s}=u_{z}^{f}=0$, which can be rewritten

$$
\begin{aligned}
\alpha_{1}[ & \left.a_{1}^{+} \exp \left(i \alpha_{1} l\right)-a_{1}^{-} \exp \left(-i \alpha_{1} l\right)\right]+\alpha_{2}\left[a_{2}^{+} \exp \left(i \alpha_{2} l\right)\right. \\
& \left.\quad-a_{2}^{-} \exp \left(-i \alpha_{2} l\right)\right]+\xi\left[a_{3}^{+} \exp (i \xi l)+a_{3}^{-} \exp (-i \xi l)\right] \\
= & 0, \\
\xi\left[a_{1}^{+}\right. & \left.\exp \left(i \alpha_{1} l\right)+a_{1}^{-} \exp \left(-i \alpha_{1} l\right)\right]+\xi\left[a_{2}^{+} \exp \left(i \alpha_{2} l\right)\right. \\
& \left.+a_{2}^{-} \exp \left(-i \alpha_{2} l\right)\right]-\alpha_{3}\left[a_{3}^{+} \exp (i \xi l)-a_{3}^{-} \exp (-i \xi l)\right] \\
= & 0, \\
\alpha_{1} \mu_{1} & {\left[a_{1}^{+} \exp \left(i \alpha_{1} l\right)-a_{1}^{-} \exp \left(-i \alpha_{1} l\right)\right] } \\
& +\alpha_{2} \mu_{2}\left[a_{2}^{+} \exp \left(i \alpha_{2} l\right)-a_{2}^{-} \exp \left(-i \alpha_{2} l\right)\right] \\
& +\xi \mu_{3}\left[a_{3}^{+} \exp (i \xi l)+a_{3}^{-} \exp (-i \xi l)\right]=0 .
\end{aligned}
$$

Let $p$ and $U_{z}$ be the pressure and the $z$ component of the air velocity in the free air at the contact surface with the porous layer. At this interface, the boundary conditions can be written ${ }^{19}$

$$
\begin{aligned}
& \phi u_{z}^{f}+(1-\phi) u_{z}^{s}=U_{z}, \\
& \sigma_{z z}^{f}=-\phi p, \\
& \sigma_{z z}^{s}=-(1-\phi) p, \\
& \sigma_{x z}^{s}=0 .
\end{aligned}
$$

A preliminary use of Eqs. (35)-(37) provides the reflection coefficients at a rigid and impervious boundary for the three Biot waves and first information concerning the shear modes. For $a_{1}^{+}=1, a_{2}^{+}=a_{3}^{+}=0$, i.e., a compressional wave $\varphi_{1}^{+}$of amplitude unity at the air-porous layer boundary, the expressions for the $a_{i}^{-}, i=1,3$, solutions of Eqs. (35)-(37) and denoted as $r_{1, i}$ are given in the first line of Table I. In the same way, the second line gives the expressions $r_{2, i}, i=1,3$, of the $a_{i}^{-}$related to $a_{2}^{+}=1, a_{1}^{+}=a_{3}^{+}=0$, and the third line gives the expressions $r_{3, i}, i=1,3$, of the $a_{i}^{-}$related to $a_{3}^{+}$ 
TABLE I. The coefficients $r_{i, j}$ expressed with $A=\alpha_{1} \alpha_{2}\left(\mu_{1}-\mu_{2}\right), B$ $=\alpha_{1} \xi\left(\mu_{1}-\mu_{3}\right), \quad C=\alpha_{2} \xi\left(\mu_{2}-\mu_{3}\right), \quad D=\alpha_{3} A+\xi(B-C), \quad$ and $\quad T_{i, j}$ $=\exp \left(-i\left(\alpha_{i}+\alpha_{j}\right) l\right)$

\begin{tabular}{lcc}
\hline \hline$r_{1,1} D T_{1,1}$ & $r_{1,2} D T_{1,2}$ & $r_{1,3} D T_{1,3}$ \\
$\alpha_{3} A+\xi(B+C)$ & $-2 \xi B$ & $-2 \xi A$ \\
$r_{2,1} D T_{2,1}$ & $r_{2,2} D T_{2,2}$ & $r_{2,3} D T_{2,3}$ \\
$2 \xi C$ & $\alpha_{3} A-\xi(B+C)$ & $-2 \xi A$ \\
$r_{3,1} D T_{3,1}$ & $r_{3,2} D T_{3,2}$ & $r_{3,3} D T_{3,3}$ \\
$-2 \alpha_{3} C$ & $2 \alpha_{3} B$ & $\alpha_{3} A-\xi(B-C)$ \\
\hline \hline
\end{tabular}

$=1, a_{1}^{+}=a_{2}^{+}=0$. The factor $\exp (i \xi x)$ has been removed from the different amplitudes. At the air-porous layer interface, each incident wave in the layer will be associated with its reflected field, so that Eqs. (35)-(37) will be automatically satisfied. Equations (38)-(41) can be rewritten

$$
\begin{aligned}
& i \alpha_{1}\left(\phi \mu_{1}+(1-\phi)\right)\left[a_{1}^{+}\left(1-r_{1,1}\right)-a_{2}^{+} r_{2,1}-a_{3}^{+} r_{3,1}\right] \\
& \quad+i \alpha_{2}\left(\phi \mu_{2}+(1-\phi)\right)\left[-a_{1}^{+} r_{1,2}+a_{2}^{+}\left(1-r_{2,2}\right)-a_{3}^{+} r_{3,2}\right] \\
& \quad+i \xi\left(\phi \mu_{3}+(1-\phi)\right)\left[a_{1}^{+} r_{1,3}+a_{2}^{+} r_{2,3}\right. \\
& \left.\quad+a_{3}^{+}\left(1+r_{3,3}\right)\right]=-i \omega U_{z}, \\
& \frac{-i}{\omega}\left\{\left(Q+R \mu_{1}\right) k_{1}^{2}\left[a_{1}^{+}\left(1+r_{1,1}\right)+a_{2}^{+} r_{2,1}+a_{3}^{+} r_{3,1}\right]+(Q\right. \\
& \left.\left.\quad+R \mu_{2}\right) k_{2}^{2}\left[a_{1}^{+} r_{1,2}+a_{2}^{+}\left(1+r_{2,2}\right)+a_{3}^{+} r_{3,2}\right]\right\}=-\phi p,
\end{aligned}
$$

$$
\begin{aligned}
& \frac{-i}{\omega}\{[\left.\left(P+Q \mu_{1}\right) k_{1}^{2}-2 N \xi^{2}\right]\left[a_{1}^{+}\left(1+r_{1,1}\right)+a_{2}^{+} r_{2,1}+a_{3}^{+} r_{3,1}\right] \\
&+\left[\left(P+Q \mu_{2}\right) k_{2}^{2}-2 N \xi^{2}\right]\left[a_{1}^{+} r_{1,2}+a_{2}^{+}\left(1+r_{2,2}\right)\right. \\
&\left.\left.+a_{3}^{+} r_{3,2}\right]+2 N \xi \alpha_{3}\left[-a_{1}^{+} r_{1,3}-a_{2}^{+} r_{2,3}+a_{3}^{+}\left(1-r_{3,3}\right)\right]\right\} \\
&=-(1-\phi) p, \\
& 2 \xi \alpha_{1}\left[a_{1}^{+}\left(1-r_{1,1}\right)-a_{2}^{+} r_{2,1}-a_{3}^{+} r_{3,1}\right] \\
& \quad+2 \xi \alpha_{2}\left[-a_{1}^{+} r_{1,2}+a_{2}^{+}\left(1-r_{2,2}\right)-a_{3}^{+} r_{3,2}\right]+\left(\xi^{2}-\alpha_{3}^{2}\right) \\
& \quad \times\left[a_{1}^{+} r_{1,3}+a_{2}^{+} r_{2,3}+a_{3}^{+}\left(1+r_{3,3}\right)\right]=0 .
\end{aligned}
$$

The three Eqs. (43)-(45) provide the ratios $a_{1}^{+} / p, a_{2}^{+} / p$, and $a_{3}^{+} / p$. Reporting these ratios in Eq. (42) provides the surface impedance $Z_{s}(\theta)=p / U_{z}$. The predicted $\cos \theta_{p}$ is obtained from the series $\left(\cos \theta_{p}\right)_{i}$ defined by the following iterative equation

$$
\left(\cos \theta_{p}\right)_{i+1}=0.8\left(\cos \theta_{p}\right)_{i}-0.2 \frac{Z}{Z_{s}\left(\left(\theta_{p}\right)_{i}\right)},
$$

with $\left(\theta_{p}\right)_{1}=\pi / 2$. A general description of the shear mode with Eqs. (42)-(45) is beyond the aim of the present work. The main properties of the mode are shown in the context of a simulation with a porous foam.

The porous material used for the simulations is a typical foam with a thickness $l=1 \mathrm{~cm}$. The parameters that describe the foam are given in Table II (yellow foam), except the imaginary part of the shear modulus $N$, and the thickness $l$. The layer is set on a rigid impervious backing and the frame

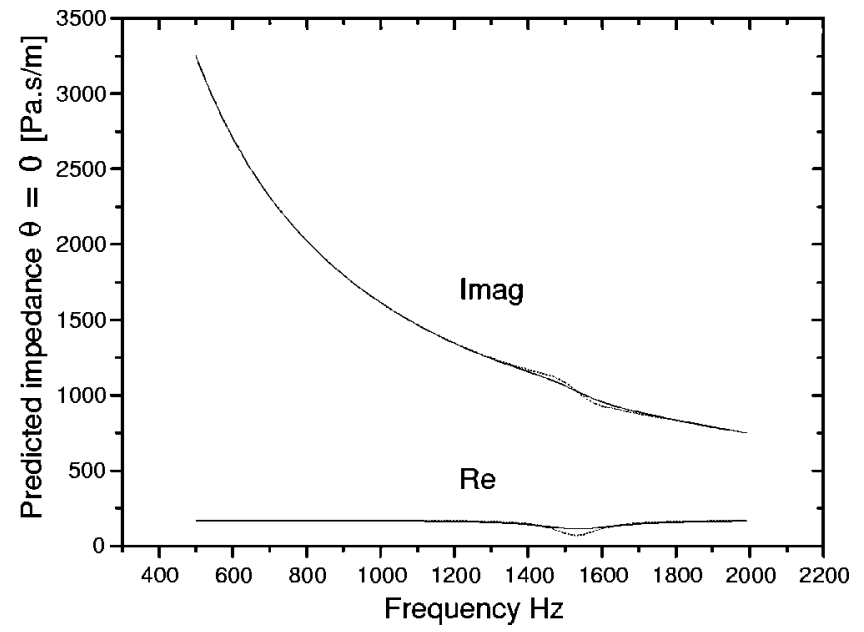

FIG. 3. The surface impedance at normal incidence. Same parameters as in the second column of Table I (yellow foam) except thickness $l=1 \mathrm{~cm}$ and $\operatorname{Im} N=-6.2 \mathrm{kPa}: \longrightarrow, \operatorname{Im} N=-1.55 \mathrm{kPa}:---$

is motionless at the contact surface. The surface impedances at normal incidence $Z_{s}(0)$ and $\cos \left(\theta_{p}\right)$ are represented in Figs. 3 and 4 for two loss angles, 0.1 and 0.025 , respectively. The Poisson ratio $\nu$ is real, because previous measurements ${ }^{7,20}$ give $\operatorname{Im} \nu=0$, and $\operatorname{Re} \nu=0.3$. This choice for $\operatorname{Re} \nu$ is arbitrary; coefficients $\nu$ previously measured for different foams range from 0.1 to 0.5 , but it has no significant effect on the shear mode. There is only a small shift, around $10 \mathrm{~Hz}$, of the location of the peak related to the shear mode in Fig. 4 when $\nu$ varies from 0 to 0.5 . The shear mode is located around $813 \mathrm{~Hz}$, where the quarter shear Biot wavelength is equal to the thickness of the layer, and the compressional mode is located around $1500 \mathrm{~Hz}$. At normal incidence, $\xi=0$, and $r_{3,1}=r_{3,2}=r_{1,3}=r_{2,3}=0, r_{3,3}=1$. Then Eq. (45) gives $a_{3}^{+}=0$ and the Biot shear wave is not present. Let $\xi_{p}$ be the horizontal wave number component related to the pole, $\xi_{p}=k \sin \theta_{p}$. When $\cos \theta_{p}$ is small, $\sin \theta_{p}$ is close to 1 , and $\xi_{p}$ is close to $k$. All the coefficients $r_{i, j}$ are different from 0 , and the shear wave is present and gives a contribution to $U_{z}$ and $p$ via $u_{z}^{s}, u_{z}^{f}, \sigma_{z z}^{s}$, and $\sigma_{z z}^{f}$. This contribution and $a_{3}^{+}$ reach a maximum when the coefficient $T_{3,3}=\exp \left(-2 i \alpha_{3} l\right)$ of

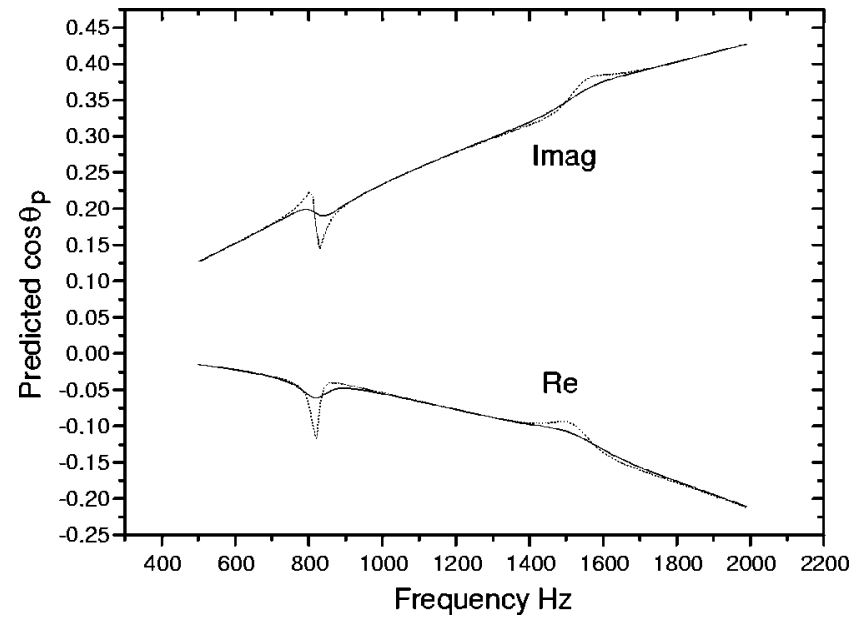

FIG. 4. Predicted $\cos \theta_{p}$ as a function of frequency for the same material as in Fig. 3. $\operatorname{Im} N=-6.2 \mathrm{kPa}: \longrightarrow, \operatorname{Im} N=-1.55 \mathrm{kPa}$ : ---. 
Table I is close to -1 , for $\operatorname{Re} \alpha_{3} l$ close to $\pi / 2$. The shear mode does not exist if the shear wave is faster than the sound in air, because in this case $\alpha_{3}=\left(k_{3}^{2}-k^{2}\right)^{1 / 2}$ is, in a first approximation, an imaginary quantity. In the simulation (and for the usual plastic open-cell foams), the speed of the shear waves in the foam is much smaller than the sound speed in the free air, and $\alpha_{3}$ is close to $k_{3}$. For a loss angle equal to $0.1(\operatorname{Im} N=-6200 \mathrm{~Pa})$, the influence of the compressional mode is negligible on the impedance but the shear mode is detectable on the behavior of $\cos \theta_{p}$. For a loss angle equal to $0.025(\operatorname{Im} N=-1550 \mathrm{~Pa})$, the presence of the shear mode is much more obvious than the presence of the compressional mode on the pole location. The shear mode appears in a small range of frequencies where it is related to a horizontal wave number component close to $k$, which allows a strong coupling with the pressure field in the free air at grazing incidence. An opposite case is the one of the Rayleigh wave, which can propagate at the same frequencies on samples of larger thickness. The order of magnitude of the Rayleigh wave velocity is around $50 \mathrm{~m} / \mathrm{s}$, which corresponds to a horizontal wave number component seven times as large as $k$. The coupling with the field in air at grazing incidence is weak, with no noticeable effect on $\cos \theta_{p}$.

\section{VALIDITY OF THE APPROXIMATION FOR $p_{r}$ IN THE CONTEXT OF THE BIOT THEORY}

The method used to measure $\cos \theta_{p}$ for thin porous layers with a motionless frame is based on the use of Eqs. (4)-(6). These expressions have been obtained with the reference integral method described by Brekhovskikh and Godin $^{21}$ from the Sommerfeld representation of the reflected monopole field

$$
p_{r}=i \int_{0}^{\infty} \frac{k \xi d \xi}{\lambda} V(\lambda) J_{0}(\xi k r) \exp \left[i k \lambda\left(h_{1}+h_{2}\right)\right],
$$

which can be rewritten

$$
\begin{aligned}
& p_{r}=\frac{i}{2} \int_{-\infty}^{\infty} \frac{k \xi d \xi}{\lambda} V(\lambda) H_{0}^{(1)}(\xi k r) \exp \left[i k \lambda\left(h_{1}+h_{2}\right)\right], \\
& \lambda=\left(1-\xi^{2}\right)^{1 / 2}, \quad \operatorname{Im}(\lambda) \geqslant 0,
\end{aligned}
$$

where $H_{0}^{(1)}$ is the Hankel function of zeroth order. A complex angle $\theta$ can be defined by $\sin \theta=\xi, \cos \theta=\lambda$. Using the asymptotic development of the Hankel function, $H_{0}^{(1)}(u)$ $=(2 / \pi u)^{1 / 2} \exp (i u-i \pi / 4)$, Eq. (48) can be rewritten

$$
\begin{aligned}
& p_{r}=\left(\frac{k}{2 \pi r}\right)^{1 / 2} \exp \left(\frac{i \pi}{4}\right) \int_{-\infty}^{\infty} F(\xi) \exp [\rho f(\lambda)] d \xi, \\
& F(\xi)=\sqrt{\frac{\xi}{1-\xi^{2}}} V(\lambda) \\
& f(\lambda)=i \cos \left(\theta-\theta_{0}\right) \\
& \rho=k R_{1} .
\end{aligned}
$$

Using the reference integral method with the notations of Ref. 21, $q=\sin \theta, q_{p}=\sin \theta_{p}$, and $q_{0}=\sin \theta_{0}, p_{r}$ can be rewritten

$$
\begin{aligned}
& p_{r}=\left(\frac{k}{2 \pi r}\right)^{1 / 2} \exp (i \pi / 4) \exp \left[\rho f\left(q_{0}\right)\right] \\
& \times\left[a \mathcal{F}_{1}\left(1, \rho, s_{p}\right)+\left(\frac{\pi}{\rho}\right)^{1 / 2} \Phi_{1}^{0}(0)\right], \\
& a=1 /\left(\frac{d F^{-1}(q)}{d q}\right)_{q=q_{p}}, \\
& \mathcal{F}_{1}\left(1, \rho, s_{p}\right)=i \pi \exp \left(-\rho s_{p}^{2}\right) \operatorname{erfc}\left(-i \sqrt{\rho} s_{p}\right), \\
& \Phi_{1}^{0}(0)=F\left(q_{0}\right)\left(-2 / f_{q^{2}}^{\prime \prime}\left(q_{0}\right)\right)^{1 / 2}+\frac{a}{s_{p}}, \\
& s_{p}=\exp (i \pi / 4) \sqrt{2} \sin \frac{\theta_{p}-\theta_{0}}{2} .
\end{aligned}
$$

With $f_{q^{2}}^{\prime \prime}\left(q_{0}\right)=-i / \cos ^{2}\left(\theta_{0}\right)$, the term $F\left(q_{0}\right)(-2 /$ $\left.f_{q^{2}}^{\prime \prime}\left(q_{0}\right)\right)^{1 / 2}$ gives a contribution $p_{r 1}$ to $p_{r}$ given by

$$
p_{r 1}=V\left(\cos \theta_{0}\right) \exp \left(i k R_{1}\right) / R_{1},
$$

independently on the expression which defines $V$. The two terms with the factor $a$ give the contribution $p_{r 2}$ given by

$$
\begin{aligned}
p_{r 2}= & a \exp \left(i k R_{1}+i 3 \pi / 4\right)(k / 2 r)^{1 / 2}\left(-i+w \sqrt{\pi} \exp \left(-w^{2}\right)\right. \\
& \times \operatorname{erfc}(-i w)) / w,
\end{aligned}
$$

where $w$ is given by Eq. (5), and the coefficient $a$ is given by

$$
\begin{aligned}
a= & \frac{-2 \cos \theta_{p}}{\sqrt{\sin \theta_{p}}}\left[1+\left(\cos \theta_{p} / Z_{s}\left(\cos \theta_{p}\right)\right)\right. \\
& \left.\times\left(d Z_{s} / d \cos \theta\right)_{\theta=\theta_{p}}\right]^{-1} .
\end{aligned}
$$

The reflected pressure can be written

$$
\begin{aligned}
p_{r}= & V\left(\cos \theta_{0}\right) \exp \left(i k R_{1}\right) / R_{1}-\frac{\cos \theta_{p}}{w} \sqrt{\frac{2 k}{r \sin \theta_{p}}} \\
& \times \frac{\exp \left(i k R_{1}+i 3 \pi / 4\right)\left[-i+w \sqrt{\pi} \exp \left(-w^{2}\right) \operatorname{erfc}(-i w)\right]}{\left[1+\left(\cos \theta_{p} / Z_{s}\left(\cos \theta_{p}\right)\right)\left(d Z_{s} / d \cos \theta\right)_{\theta=\theta_{p}}\right]} .
\end{aligned}
$$

If $Z_{s}$ does not depend on $\theta_{p}$, this equation is identical to Eq. 1-4-10 of Ref. 21 for locally reacting surfaces

$$
\begin{aligned}
p_{r}= & V\left(\cos \theta_{0}\right) \exp \left(i k R_{1}\right) / R_{1}-\frac{\cos \theta_{p}}{w} \sqrt{\frac{2 k}{r \sin \theta_{p}}} \exp \left(i k R_{1}\right. \\
& +i 3 \pi / 4)\left[-i+w \sqrt{\pi} \exp \left(-w^{2}\right) \operatorname{erfc}(-i w)\right] .
\end{aligned}
$$

If $\theta_{p}$ and $\theta_{0}$ are sufficiently close to $\pi / 2,\left(\sin \theta_{p}\right)^{1 / 2}$ can be removed and Eqs. (5) and (62) can be replaced by Eqs. (6) and (4). These equations can be used also for thin layers with a motionless frame or in the context of the Biot theory if

$$
\left|\left(\cos \theta_{p} / Z_{s}\left(\cos \theta_{p}\right)\right)\left(d Z_{s} / d \cos \theta\right)_{\theta=\theta_{p}}\right| \ll 1 .
$$

An evaluation of the left side of this equation is not very simple. If the structural damping of the frame is small, the shear mode induces fast variations of $Z_{s}$ with frequency, Eq. (63) is not satisfied, and Eqs. (4)-(6) cannot be used, but the loss angle for usual open-cell foams is large. Comparisons 


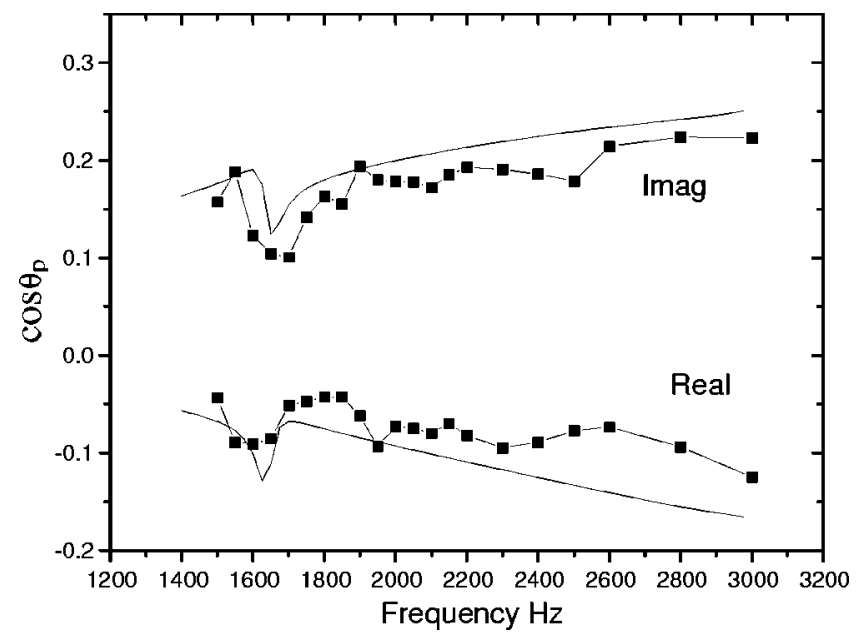

FIG. 5. $\cos \theta_{p}$ as a function of frequency, yellow foam; measurement $\mathbf{\square}-\mathbf{\square}$, prediction $\longrightarrow$

between estimations of the reflected pressure over thin porous layers, obtained with Eq. (47) and with Eqs. (4)-(6), have been performed around frequencies where the shear mode is present, showing that for loss angles of the frame larger than $\frac{1}{100}$, Eqs. (4) and (6) provide a good approximation for the reflected pressure for thin layers. The loss angle for usual open-cell foams is much larger than $\frac{1}{100}$.

\section{EXPERIMENTAL EVALUATION OF THE SHEAR MODULUS}

In Fig. 1 a symbolic sketch of the experimental set is presented. The monopole source $\mathrm{S}$ is a pipe fed by a compression driver. The receiver at $\mathrm{M}$ is an electret microphone. The distances $z_{1}$ from the porous layer to the source and $z_{2}$ from the layer to the microphone are $z_{1}=z_{2}=3 \mathrm{~mm}$. The distance $r$ from the source to the receiver is $r=23 \mathrm{~cm}$. The angle of specular reflection is $\theta_{0}$ verifying $\cot \theta_{0}=\left(z_{1}\right.$ $\left.+z_{2}\right) / r$. The porous layers are glued to a rigid impervious backing, the area of the layers is close to $1 \mathrm{~m}^{2}$. The evaluation of $\cos \theta_{p}$ with Eq. (4) is performed from a measurement of the ratio $p_{r} /\left(\exp \left(i k R_{1}\right) / R_{1}\right)$ where $\exp \left(i k R_{1}\right) / R_{1}$ is the direct field at a distance $R_{1}$ from the source. The measurement of the ratio is obtained from a measurement of the pressure $p_{h}$ related to the direct field, the porous surface being replaced by a rigid, impervious surface and a second measurement over the porous surface of the pressure $p_{t}$. Both measurements are performed with the same geometry, close to grazing incidence so that $R_{1}$ and $R_{2}$ are equal in a first approximation. The ratio in Eq. (4) can be evaluated from

$$
p_{r} /\left(\exp \left(i k R_{1}\right) / R_{1}\right)=\frac{p_{t}-p_{h} / 2}{p_{h} / 2},
$$

and $\cos \theta_{p}$ is given by

$$
\begin{aligned}
2 \frac{p_{h}-p_{t}}{p_{h}}= & \cos \theta_{p} \exp \left(\frac{3 \pi i}{4}\right)\left[\sqrt{2 \pi k R_{1}} \exp \left(-w^{2}\right)\right. \\
& \times \operatorname{erfc}(-i w)] .
\end{aligned}
$$

An iterative procedure $^{14}$ is used to evaluate $\cos \theta_{p}$ from Eq. (65). For thin layers with a source-receiver

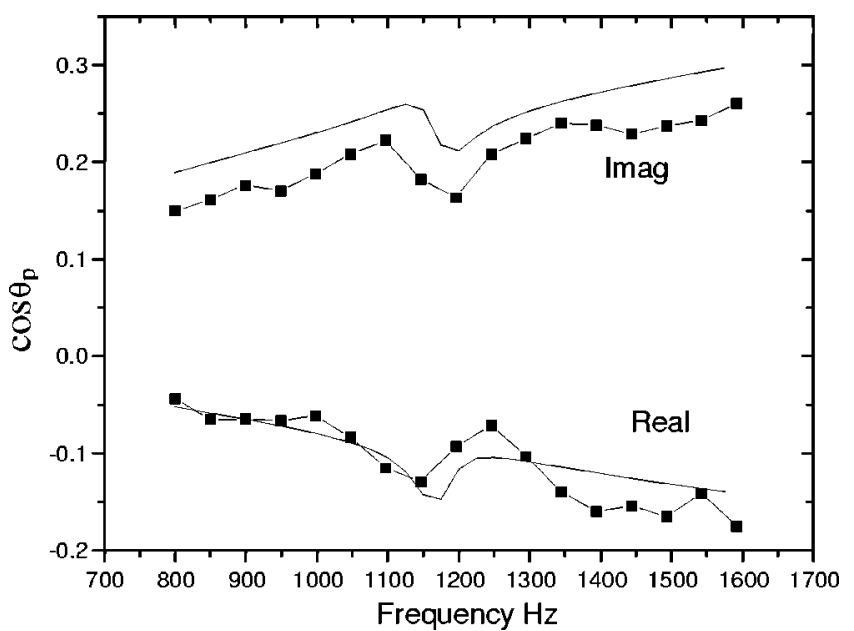

FIG. 6. $\cos \theta_{p}$ as a function of frequency, black foam; measurement - $\mathbf{\square}-\mathbf{\square}$, prediction $\longrightarrow$.

distance smaller than $1 \mathrm{~m}, w$ is small and the first value for $\cos \theta_{p}$ is obtained with $w=0$, giving $\cos \theta_{p}$ $=2\left(1-p_{t} / p_{h}\right) / \exp (3 \pi i / 4) \sqrt{2 \pi k R_{1}}$. The derivative of $g(w)$ $=\exp \left(-w^{2}\right) \operatorname{erfc}(-i w)$ is $g_{w}^{\prime}(w)=-2 w g(w)+2 i / \sqrt{\pi}$ (see Ref. 22, Chap. 7), and the derivative $d w / d \cos \theta_{p}$ of $\quad w=\left(1 / 2 i k R_{1}\right)^{1 / 2}\left(\cos \theta_{0}-\cos \theta_{p}\right) \quad$ is $\quad d w / d \cos \theta_{p}=$ $-\left(1 / 2 i k R_{1}\right)^{1 / 2}$. These two relations are used to minimize the left- and right-hand side difference of Eq. (65).

Measurements have been performed on two foams, a yellow foam of thickness $l=0.5 \mathrm{~cm}$ and a black foam (S616D) manufactured by Recticel (Plejadenlaan, 15, B-1200 Brussels) of thickness $l=1 \mathrm{~cm}$. Measurements on the yellow foam are presented in Fig. 5 and on the black foam in Fig. 6. Fast variations of $\cos \theta_{p}$ similar to the ones of Fig. 4 are present for both foams. A set of parameters given in Table II has been used to predict $\cos \theta_{p}$ in Figs. 4 and 5. The flow resistivities and the densities in Table II are measured and the shear moduli have been chosen to set the fast predicted variations at the same frequencies as the measured ones. The other parameters have been set to reasonable values. The frequencies where the fast variations occur are mainly related to densities and shear moduli; the other parameters have a very small influence on the localization of the fast variations. For the black foam, measurements of the Rayleigh wave velocity have been performed on a sample of thickness $l=6 \mathrm{~cm}$ with the method described in Ref. 8: a Rayleigh-like wave is excited with a conventional shaker in

TABLE II. Parameters for the porous materials.

\begin{tabular}{lcc}
\hline \hline Parameters & Yellow foam & Black foam \\
\hline porosity $\phi$ & 0.99 & 0.99 \\
flow resistivity $\sigma\left(\mathrm{Nm}^{-4} \mathrm{~s}\right)$ & 40000.0 & 130000.0 \\
thermal permeability $k_{0}^{\prime}\left(\mathrm{m}^{2}\right)$ & $10^{-9}$ & $5 \times 10^{-10}$ \\
viscous dimension $\Lambda(\mu \mathrm{m})$ & 100 & 60 \\
thermal dimension $\Lambda^{\prime}(\mu \mathrm{m})$ & 300 & 180 \\
tortuosity $\alpha_{\infty}$ & 1.05 & 1.1 \\
density $\rho_{s}\left(\mathrm{~kg} / \mathrm{m}^{3}\right)$ & 58 & 59 \\
shear modulus $N(\mathrm{kPa})$ & $62.0-i 1.55$ & $125.0-i 6.0$ \\
Poisson ratio $(\nu)$ & 0.3 & 0.3 \\
thickness $l(\mathrm{~cm})$ & 0.5 & 1.0 \\
\hline \hline
\end{tabular}



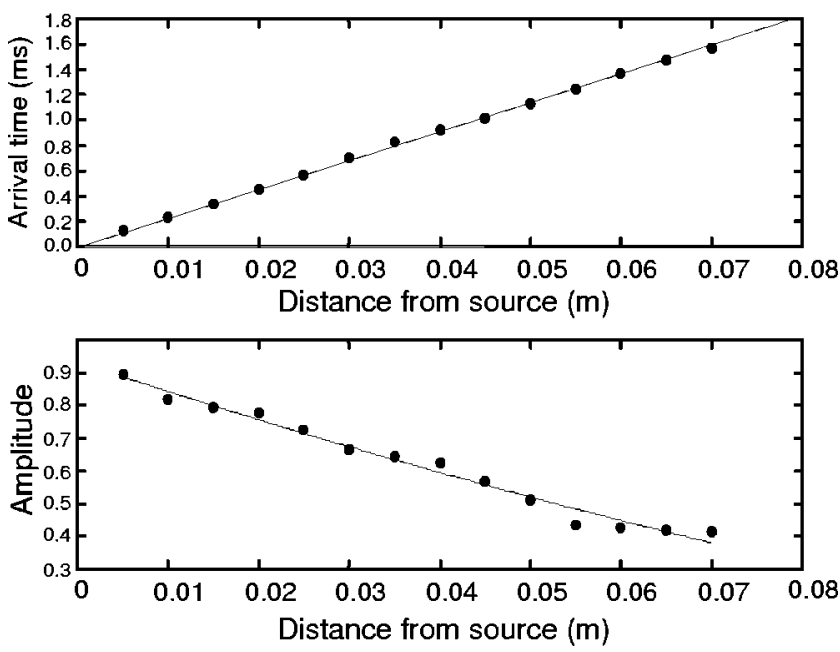

FIG. 7. Arrival time and attenuation as a function of position at $2 \mathrm{kHz}$, $25{ }^{\circ} \mathrm{C}$. The phase velocity and attenuation are determined from a linear regression and an exponential regression on the data, respectively.

contact with the surface of the sample and the vertical displacement is measured at several distances from the source using a Polytec vibrometer. A line source has been used as an excitation and the signal was a narrow-band Gaussian burst centered around the measuring frequency. The shear modulus was extracted from the phase velocity of the Rayleigh wave and the attenuation was measured from the decay of the amplitude as a function of position. Figures 7(a) and (b) show typical results as a function of position at a center frequency of $2 \mathrm{kHz}$ and ambient temperature $25^{\circ} \mathrm{C}$. Table III summarizes the results at 2 and $4 \mathrm{kHz}$ under the hypothesis of a Poisson ratio $\nu=0.3$. The yellow foam is much less rigid at very low frequencies than the black foam, but it has not been possible to measure the Rayleigh wave velocity (samples of sufficient thickness were not available). The effect of the shear mode is a peak limited over a small range of frequencies. The precision of the measurement of $\operatorname{Re} N$ is limited by the broadness of the peak. For the materials studied, the precision is about $\frac{10}{100}$ for $\operatorname{Re} N$ and $\frac{50}{100}$ for $\operatorname{Im} N$. The fast increase of $\operatorname{Im} N$ with frequency can explain the difference between the measured $\operatorname{Im} N$ around $1.2 \mathrm{kHz}$ with the point source method and around $2 \mathrm{kHz}$ with the Rayleigh wave. The measurements of $\operatorname{Re} N$ by both methods give similar results.

\section{CONCLUSION}

The acoustic field created by a point-source close to thin layers of open-cell foams can be strongly coupled with a shear mode around frequencies where the quarter shear wavelength is equal to the thickness of the layer. The strong coupling is due to the fact that the horizontal wave-number

TABLE III. Measured wave number of the Rayleigh wave and shear modulus.

\begin{tabular}{cccc}
\hline \hline $\begin{array}{c}\text { Frequency } \\
(\mathrm{kHz})\end{array}$ & $\begin{array}{c}\operatorname{Re} k_{R} \\
\left(\mathrm{~m}^{-1}\right)\end{array}$ & $\begin{array}{c}\operatorname{Im} k_{R} \\
\left(\mathrm{~m}^{-1}\right)\end{array}$ & $\begin{array}{c}N \\
(\mathrm{kPa})\end{array}$ \\
\hline 2 & 283.0 & 12.0 & $135.0-i 11.0$ \\
4 & 576.0 & 65.0 & $126.0-i 29.0$ \\
\hline \hline
\end{tabular}

component of the mode is close to the wave number in the free air. The amplitude of the mode is sufficiently large for the mode to be detected acoustically from the fast variations with frequency of the location of a pole of the reflection coefficient. The description with the Biot theory of the interaction between the shear mode and the acoustic field in air is in good agreement with the experiment. This is a new example of the adequacy of the Biot theory for the description of the acoustical properties of sound-absorbing media. This work also shows the interest of the method of localizing a pole of the reflection coefficient from short range pressure measurements close to grazing incidence.

${ }^{1}$ T. Pritz, "Transfer function method for investigating the complex modulus of acoustic materials: spring-like specimen," J. Sound Vib. 72, 317-341 (1980).

${ }^{2}$ T. Pritz, "Transfer function method for investigating the complex modulus of acoustic materials: rod-like specimen," J. Sound Vib. 81, 359-376 (1982).

${ }^{3}$ T. Pritz, "Frequency dependence of frame dynamic characteristics of mineral and glass wool materials," J. Sound Vib. 106, 161-169 (1986).

${ }^{4}$ T. Pritz, "Dynamic Young's modulus and loss factor of plastic foams for impact sound isolation," J. Sound Vib. 178, 315-322 (1994).

${ }^{5}$ A. Sfaoui, "On the viscoelasticity of the polyurethane foam," J. Acoust. Soc. Am. 97, 1046-1052 (1995).

${ }^{6} \mathrm{~T}$. Pritz, "Measurement methods of complex Poisson's ratio of viscoelastic materials," Appl. Acoust. 60, 279-292 (2000).

${ }^{7}$ M. Melon, M. Mariez, C. Ayrault, and S. Sahraoui, "Acoustical and mechanical characterization of anisotropic open-cell foams," J. Acoust. Soc. Am. 104, 2622-2627 (1998).

${ }^{8}$ J. F. Allard, G. Jansens, G. Vermeir, and W. Lauriks, "Frame-borne surface waves in air-saturated porous media," J. Acoust. Soc. Am. 111, 690696 (2002).

${ }^{9}$ M. A. Biot, "Theory of propagation of elastic waves in a fluid-saturated porous solid," J. Acoust. Soc. Am. 28, 168-191 (1956).

${ }^{10} \mathrm{M}$. A. Biot and D. G. Willis, "The elastic coefficients of the theory of consolidation," J. Appl. Mech. 24, 594-601 (1957).

${ }^{11}$ J. F. Allard, C. Depollier, P. Guignouard, and P. Rebillard, "Effect of a resonance of the frame on the surface impedance of a glass-wool of high density and stiffness," J. Acoust. Soc. Am. 89, 999-1001 (1991).

${ }^{12}$ M. Tamura, "Spatial Fourier transform method of measuring reflection coefficient at oblique incidence," J. Acoust. Soc. Am. 88, 2259-2264 (1990)

${ }^{13}$ J. F. Allard, M. Henry, V. Gareton, J. Jansens, and W. Lauriks, "Impedance measurement around grazing incidence for nonlocally reacting thin porous layers," J. Acoust. Soc. Am. 113, 1210-1215 (2003).

${ }^{14}$ J. F. Allard, V. Gareton, M. Henry, G. Jansens, and W. Lauriks, "Impedance evaluation from pressure measurements near grazing incidence for nonlocally reacting porous layers," Acust. Acta Acust. 89, 595-603 (2003)

${ }^{15}$ J. F. Allard, M. Henry, and V. Gareton, "Pseudo-surface waves above thin porous layers," J. Acoust. Soc. Am. 116, 1345-1347 (2004).

${ }^{16}$ J. F. Allard, Propagation of Sound in Porous Media, Modelling Sound Absorbing Materials (Elsevier, London, 1993).

${ }^{17}$ D. L. Johnson, J. Koplik, and R. Dashen, "Theory of dynamic permeability and tortuosity in fluid-saturated porous media," J. Fluid Mech. 176, 379-402 (1987)

${ }^{18}$ D. Lafarge, P. Lemarinier, J. F. Allard, and V. Tarnow, "Dynamic compressibility of air in porous structures at audible frequencies," J. Acoust. Soc. Am. 102, 1995-2006 (1997).

${ }^{19}$ H. Deresiewicz and R. Skalak, "On uniqueness in dynamic poroelasticity," Bull. Seismol. Soc. Am. 53, 783-788 (1963).

${ }^{20}$ E. Mariez, S. Sahraoui, and J. F. Allard, "Elastic constants of polyurethane foam's skeleton for Biot model," Internoise Congress Proceedings, Budapest (1997), pp. 1683-1686.

${ }^{21}$ L. M. Brekhovskikh and O. A. Godin, Acoustics of Layered Media II, Point Source and Bounded Beams, Springer Series on Wave Phenomena (Springer, New York, 1992).

${ }^{22}$ M. Abramovitz and I. A. Stegun, Handbook of Mathematical Functions (Dover, New York, 1970). 